\title{
Simultaneous Flow and Thermal Conductivity Sensing on a Single Chip using Artificial Neural Networks
}

\author{
Ethan L.W. Gardner ${ }^{1,2}$, Timothy A. Vincent ${ }^{2}$, Andrea De Luca ${ }^{1,2}$, Florin Udrea ${ }^{1,2}$ \\ ${ }^{1}$ University of Cambridge, Cambridge, United Kingdom \\ ${ }^{2}$ Flusso Ltd., Cambridge, United Kingdom
}

\begin{abstract}
A thin-film CMOS MEMS thermal sensor has been designed, fabricated and tested with the addition of through-membrane isolating holes. These holes have been shown to enhance the discrimination towards gases with differing thermal conductivity in the presence of flow. Using three on-membrane resistors as inputs, linear statistical methods alongside Artificial Neural Network pattern recognition techniques have been investigated for decoupling the two parameters of thermal conductivity and flow rate using a single sensor. In addition to this, the addition of the membrane holes increases the sensitivity towards flow rate by 10 times and the sensitivity towards thermal conductivity by 2 times. This sensor design coupled with well-known post-processing techniques will enable a new generation of multi-parameter sensing solutions.
\end{abstract}

Keywords-MEMS; flow sensor; thermal conductivity sensor; micro-fabrication; artificial neural networks.

\section{INTRODUCTION}

With the discovery and wide-spread use of micromachining techniques, thermal based sensors for the measurement of physical phenomena have found themselves in high demand across a multitude of industrial applications. This is thanks to their ability of implementation in a wide range of applications such as flow, thermal conductivity, diffusivity, heat capacity and pressure, among others. With their commonplace and continued research, these devices are becoming smaller, cheaper, more power efficient, more sensitive, more accurate and smarter.

There has been extensive research on the development of micromachined flow sensors and a detailed review can be found here [1]. In addition to this, micromachined flow sensors can be used to detect thermal properties of fluids such as thermal conductivity and thermal diffusivity using transient excitation such as the $3 \omega$ method [2], [3]. Simultaneous thermal conductivity and thermal diffusivity sensing has also been shown [4].

Using micromachined thermal sensors for the measurement of thermal conductivity has attracted recent attention over their more researched counterparts of calorimetric or metal oxide gas sensors. This is due to their ability to take measurements more quickly and that they do not rely on a reaction with a catalyst. There has been work looking to exploit the benefits of these sensors for gas chromatography [5], and for gas discrimination through DC and transient excitation [6], [7]. Despite these recent advancements, there is still little research into solving the problem of dual flow and thermal conductivity sensing, due to the high interdependence of both parameters on sensor response. This has been investigated by combing results from several sensors in conjunction with complicated data acquisition [8]. [9] used a calorimetric flow sensor to measure thermal gas properties in non-stagnant flow. However, this method only works in previously calculated regions of flow independence.

The fabricated devices in this report incorporate holes through the membrane on either side of the resistor that isolate the resistor. This new design provides multiple benefits that include: (i) the ability to negate front and backside pressure differences, resulting in a large reduction in the number of catastrophic membrane breakages during fabrication and, especially, packaging, hence increasing through yield of the final device, (ii) minimising conductive heat losses through the membrane, thus improving the electro-thermal efficiency and power consumption of the device, (iii) minimising conduction through the membrane which also forces more interaction between the measuring resistors and the fluid flowing, and (iv) creating an opening to the cavity below the membrane, resulting in twice the exposure to the gas due exposure and thermal heat losses from both sides of the resistor.

Smart sensors and sensor systems involve so many variables that it isn't practical to seek a hard, analytical model explicitly relating all the parameters. Many such methods for providing solutions have been proposed, with particular interest and success coming from linear statistical methods, such as Principal Component Regression (PCR) and Partial Least Squares Regression (PLSR). Some examples of such techniques in order to discriminate substances from a variety of inputs include [10], [11], with an overview of the technique and its diversity in applications here [12]. Artificial Neural Networks (ANNs) are another desirable tool used for powerful pattern recognition and data discrimination. This has led to the widespread use of ANNs for solving multivariable functions, and more specifically for use with arrays of sensors. Some examples include electronic noses [13], 
[14], where a combination of metal oxide sensors are used in conjunction with one another, an optical sensor array for identifying multiple analytes [15], the use of impedance spectroscopy for the classification of wine [16], decoupling carbon monoxide and methane using platinum and tin dioxide sensors [17] and more recently, gas discrimination using a metal oxide-modified graphene-based sensor array [18].

In this work, we report on linear statistical methods Partial Least Squares Regression (PLSR) and Principal Component Analysis (PCR) as well as an ANN approach for processing signals that arise from three resistors located in the membrane of a CMOS MEMS thermal flow sensor. The thermal flow sensor incorporates isolating holes through the membrane in order to enhance the gas discrimination. These postprocessing techniques are used to de-couple the closely related parameters of thermal conductivity and flow rate. A sensor that can successfully discriminate between these parameters will be in high demand throughout industrial applications and this paper serves to pave the way for the combination of a single-membrane, low-cost, low-power sensor and smart post-processing techniques for a new generation of multi-parameter sensing solutions.

\section{EXPERIMENTAL DETAILS}

\section{A. Sensor Design \& Fabrication}

The sensors were designed using Cadence Virtuoso (IC 5.1.4) and commercially fabricated with a $1.0 \mu \mathrm{m}$ standard CMOS process. The backside of the chip was etched using Deep Reactive Ion Etching (DRIE) in order to create multiple thin-film membranes on one sensor, benefiting from enhanced thermal isolation and multiple independent sensors on one device. An example of one of the chips is shown in Figure 1 with its cross-section schematically depicted in Figure 2. For this work, two membranes on the same chip that can be run independently were investigated. Both designs include 3 parallel tungsten heating resistors of identical dimensions and separation connected to wire bonding pads with $20 \mu \mathrm{m}$ tracks. An extra processing level was incorporated in order to add holes through the thin-film membrane in one of these designs. These holes are incorporated for three reasons: (i) to reduce the chance of catastrophic breakage due to pressure difference below and above the membrane, (ii) to isolate the heating resistor and reduce losses through conduction and (iii) to force more interaction with the passing medium, i.e. increased levels of convection with the flowing gas. The two designs are called 'no holes' (reference design), and 'holes' (heating resistor isolation through holes on both sides of central resistor) where both designs can be seen in Figure 1. The 'holes' design contains 4 holes in parallel on both sides of the central heater, ensuring full isolation. All membrane holes have the same geometry of $10 \mu \mathrm{m} \times 200 \mu \mathrm{m}$ with rounded edges to avoid stress concentration factors. Using a Kulicke and Soffa wedge bonder, the sensors were bonded onto Dual in Line (DIL8) packages, raised and filled to ensure a planar surface

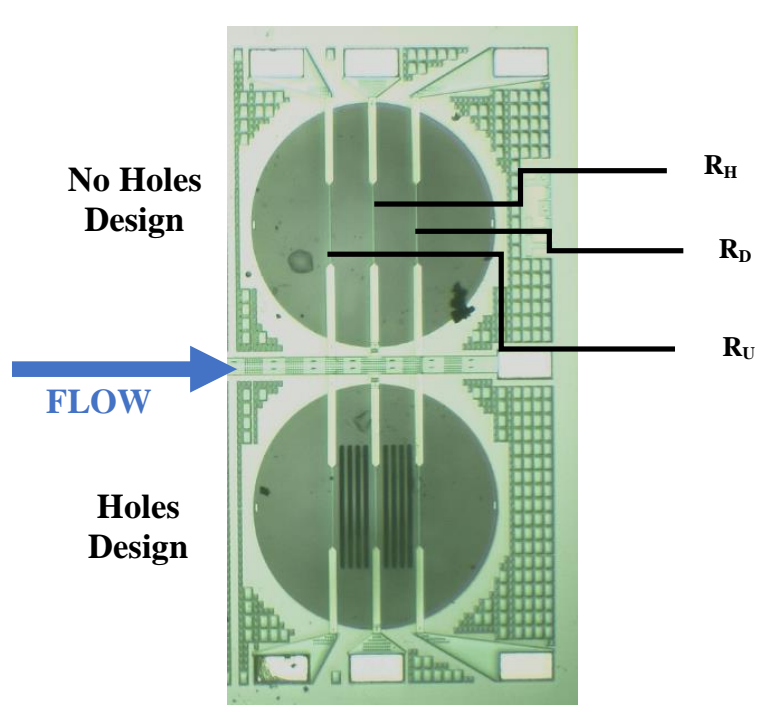

Figure 1. Optical micrograph of one of the sensor chips - die dimensions of $2.0 \mathrm{~mm} \times 2.0 \mathrm{~mm}$.

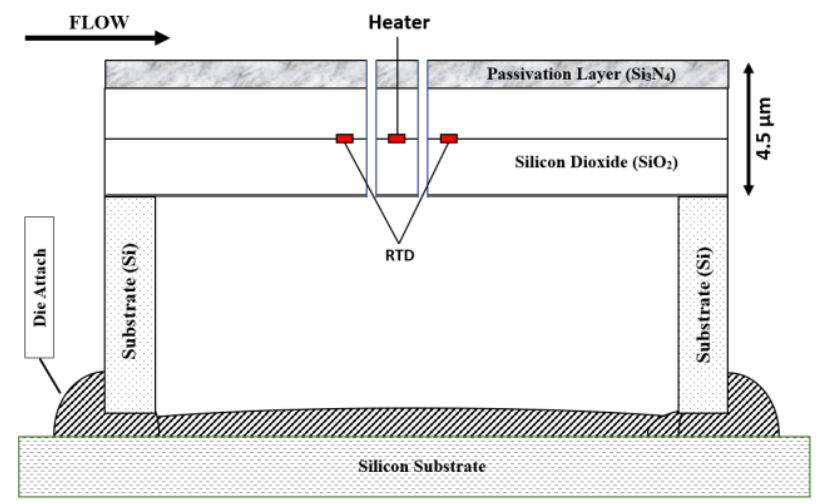

Figure 2. A cross-section of the layer technology used in fabrication for the design with membrane holes.

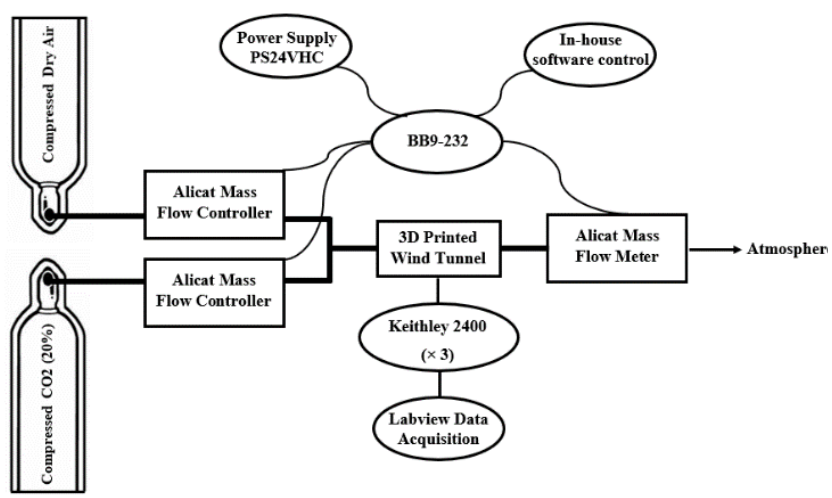

Figure 3. A schematic of the experimental setup.

and integrated into an in-house 3D-printed channel of crosssectional area $1.5 \mathrm{~mm} \times 2.5 \mathrm{~mm}$. A detailed review of the fabrication technology, sensors and their packaging can be found here [19]. 


\section{B. Measuring Setup \& Testing Protocol}

Measurements were performed in two separate experimental protocols. Firstly, the electro-thermal characterisation of the device heaters was performed between $25^{\circ} \mathrm{C}$ and $300{ }^{\circ} \mathrm{C}$ using a Cascade probe station and hot chuck with Keithley source meters for data acquisition. This enabled the collection of important material properties such as the Temperature Coefficients of Resistance (TCR) and electrothermal efficiency. For more detail on the details of electrothermal characterisation please refer to [19]. Using the information from this characterisation, the measurements with flow and $\mathrm{CO}_{2}$ were carried out using an array of Alicat Mass Flow Controllers (MFC), alongside an array of Keithley 2410 Source Meter Units (SMU) for data acquisition of the three resistors. $\mathrm{CO}_{2}$ was chosen due to its high importance and demand in consumer products relating to human health, such as air quality monitoring. A diagrammatic representation of the experimental setup is displayed in Figure 3. Each MFC was located after a gas cylinder to control the amount of $\mathrm{CO}_{2}$ and air, which was subsequently fed into a mixing valve before the flow tunnel or order to vary the $\mathrm{CO}_{2}$ concentration. Three packaged devices (three of each design) were subsequently exposed to a flow rate from 0 to 240 SCCM in steps of 40 SCCM in five different concentrations of $\mathrm{CO}_{2}$ in air $(0 \%, 5 \%, 10 \%, 15 \%, 20 \%)$. Such a flow range was chosen to ensure laminar behaviour was maintained in the channel. A bias current of $8 \mathrm{~mA}$ was applied to the central resistor for all experiments. The three resistors $R_{H}, R_{U}$ and $R_{D}$, where subscripts $H, U$ and $D$ represent the heater, upstream resistor and the downstream resistor, respectively, are measured and the change in voltage of each $V_{H}, V_{U}$ and $V_{D}$ are considered as the input variables for the post-processing techniques.

\section{Linear Statistical Methods}

Linear statistical methods were employed in order to test whether they can establish a predictive model from a set of independent variables (resistors) onto another set of continuous variables (targets). Partial Least Squares Regression (PLSR) and Principal Components Regression (PCR) are both methods that are used to model a response variable with many input variables, when the input variables are highly correlated or collinear. Both techniques construct new predictive components which are linear combinations of the original input variables, however the formation of these components is different and both methods are employed in order to see which is the most effective at mapping the behavior correctly. The main difference between the two techniques is that PCR does not consider the target variable to explain observed variability in the inputs, whereas PLSR does, thus often leading to an adequate fit in fewer components. PLSR assumes that the predictive components account for most of the variation measured by the original variables $\boldsymbol{X}_{(n, p)}$, that are linked to the response $\boldsymbol{Y}_{(n, 1)}$ through the linear relationship of $\boldsymbol{Y}=\alpha+\boldsymbol{X} \beta+\boldsymbol{\theta}$ where the unknown regression parameters are $\alpha$ and $\beta$ and $\boldsymbol{\theta}$ is the error term.

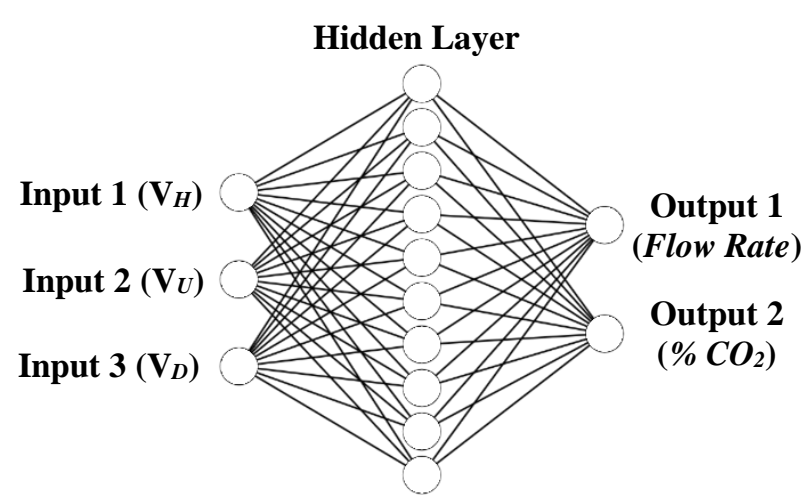

Figure 4. A diagram of the 3-layer network incorporated for 3 inputs and 2 outputs fully connected with 10 neurons in the hidden layer.

PLSR combines the properties of multiple linear regression and principal component analysis, producing a technique that separates sample noise and makes linear combinations in a dependent concentration matrix. It is widely used as the most effective linear statistical method for chemometrics and so is considered the best technique to use [20].

\section{Network Architecture}

Artificial Neural Networks (ANNs) consist of an interconnected web of parallel, adaptive elements, of which the architecture is based upon the physical biological system, namely on neurons and their interconnections, i.e. synaptic links. In our case, ANNs have been employed in order to decouple the closely related parameters of flow and thermal conductivity. A network of three layers is chosen, due to its previous success in multi-parameter gas sensing and that adopting this format has enough degrees of freedom to solve any problem [21]. This three-layer network was implemented in order to model our 3-element system, where the input layer contains 3 nodes that correspond to each of the on-membrane resistor outputs $V_{H}, V_{U}$ and $V_{D}$. Each input node is connected to 10 nodes in the hidden layer, in which the elements are to be experimentally determined during the training phase. The outer layer consists of 2 nodes that correlate to the 2 parameters which we want to identify, namely the flow rate (SCCM) and thermal conductivity $\left(\% \mathrm{CO}_{2}\right)$. The network consists of 3 input nodes that are fully connected to 10 neurons in the hidden layer, which are fully connected to the two output nodes. A graphical depiction of this network architecture can be found in Figure 4.

It is known that the learning algorithms of the nodes determine the performance of any implemented network. There are a multitude of different algorithms, and we have chosen the widely used back-propagation method. This method is chosen because it is fast, simple, flexible and requires no previous knowledge about the network, thus only needing to tune number of inputs. Above these reasons, it is used because it is the standardly used method, improving applicability and convenience. 


\section{RESULTS \& DISCUSSION}

\section{A. Response to Flow \& Thermal Conductivity}

The packaged devices were exposed to a flow rate from 0 to 240 SCCM in steps of 40 SCCM. This flow rate range was chosen due to turbulent flow being produced above this value, resulting in more heat extracted from the heater yielding an unpredictable response. The range is used to evaluate the laminar region, which could easily be expanded, for example by enlarging the flow tunnel dimensions. Figure 5 shows the enhanced gas discrimination shown between air and 20\% $\mathrm{CO}_{2}$ for the designs with and without holes for all three resistors. All values of voltage change were normalised by the base resistance by taking the voltage change with respect to no flow. It should be noted that the plots also show the absolute values of voltage change. Figure 5 (a) shows the voltage change of the heater $V_{H}$, which shows a higher sensitivity with flow as well as clear differentiation between the line of air and $20 \% \mathrm{CO}_{2}$, yielding strong discrimination between gases even with the presence of flow. Figure 5 (b) is the voltage change in the downstream resistor $V_{D}$. As the peak of the thermal profile is shifted through forced convection, the voltage change is initially negative. This effect is significantly increased with the addition of membrane holes, resulting in the increased calorimetric benefit as well as providing more identifiable and detectable features for multi parameter data analysis, specifically with regards to flow rate as this feature is solely due to fluid flow and not its thermal conductivity. Finally, Figure 5 (c) shows the upstream resistor $V_{U}$, providing another parameter with higher sensitivity to flow and better gas discrimination.

Using membrane holes has vastly increased the selectivity to thermal conductivity with the presence of flow. Using this design and all the improved gas selectivity responses, linear statistical methods and ANNs will be used to try and successfully identify and discriminate these two parameters.

\section{B. Data Pre-Processing}

It is important to examine the data that is generated by the array of three resistors in order to make the most informed choice of pre-processing method. Informed decisions can be designed to help analyze the data for our specific problem, such as compensating for fluctuations in the vectors. There were two pre-processing protocols implemented in this work:

a. The input values $V_{H}, V_{U}$ and $V_{D}$ were auto ranged in order to make their range from -1 to +1 by using the equation $\left[2\left(V-V_{M I N}\right) /\left(V_{M A X^{-}} V_{M I N}\right)-1\right]$.

b. The output values were normalized to $\mathrm{U} / \mathrm{U}_{\mathrm{Max}}$ and $\% \mathrm{CO}_{2} / \% \mathrm{CO}_{2}$, Max. Where $\mathrm{U}$ refers to the flow rate in Standard Cubic Centimeters per Minute (SCCM), $\mathrm{U}_{\mathrm{Max}}$ is the highest value of flow rate, $\% \mathrm{CO}_{2}$ is the percentage $\mathrm{CO}_{2}$ in air and $\% \mathrm{CO}_{2}$, Max is the max percentage of $\mathrm{CO}_{2}$ in air.
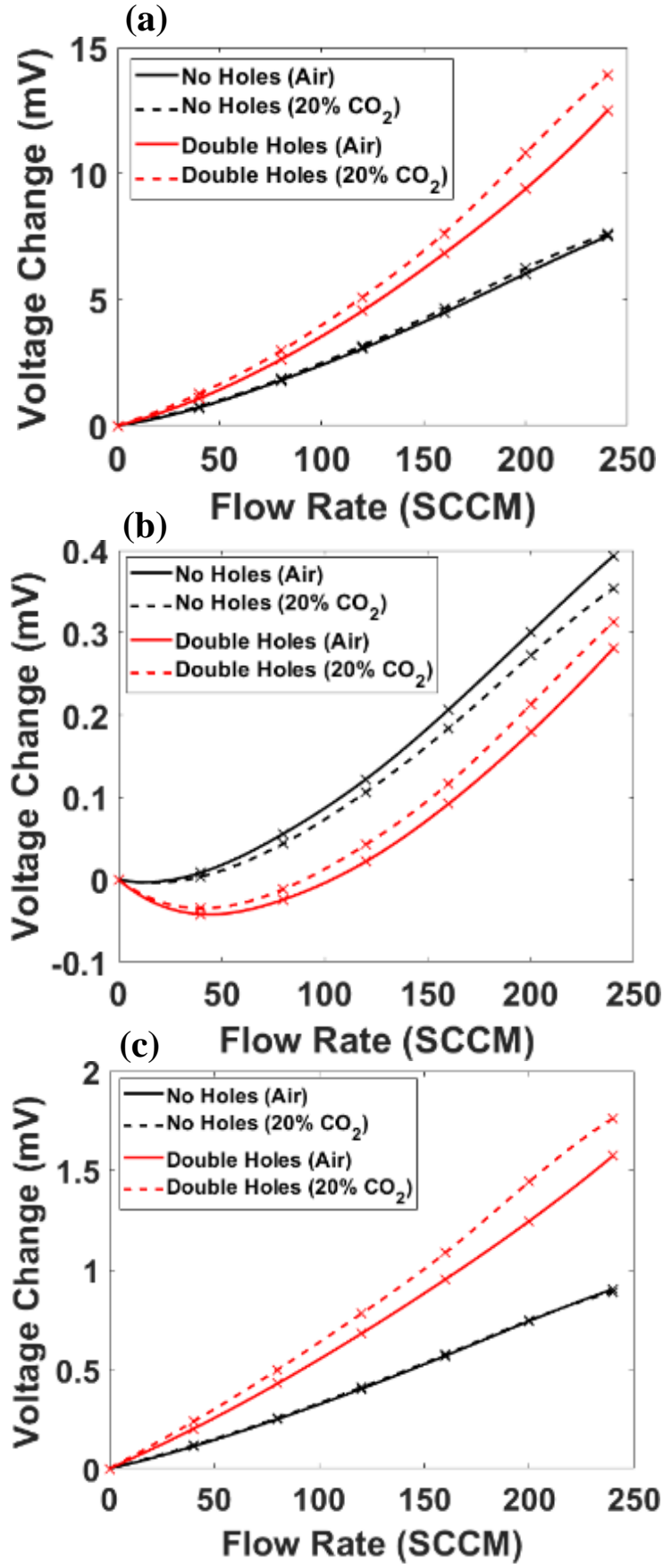

Figure 5. Three plots showing the discrimination between air and $20 \%$ $\mathrm{CO}_{2}$ after being normalised by base resistance for the designs with double holes and without holes for (a) the central resistor, (b) downstream resistor and (c) the upstream resistor.

These pre-processing techniques will help the performance of the multi-variable analysis. Auto-ranging the data means that all three sensors are made to be equal magnitude, and therefore have equal influence on the network. Auto-ranging the inputs whilst also normalizing the targets is performed to help set the weights in the neural network that will start in this range, yielding quicker and better convergence. 


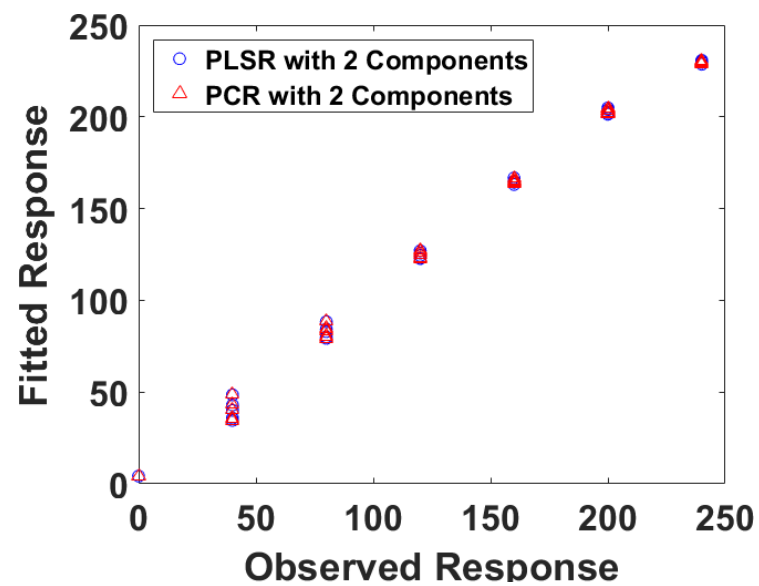

Figure 6. The observed responses of the experiment against the fitted responses from PLSR and PCR linear correlation methods for flow rate outputs.

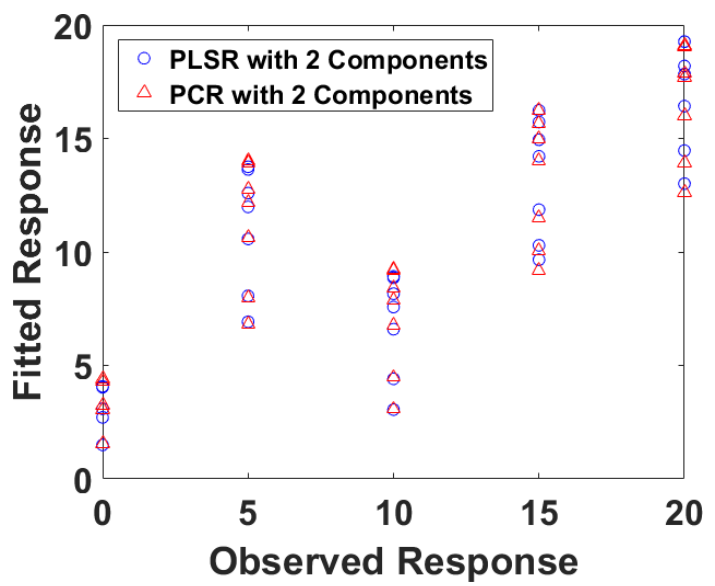

Figure 7.. The observed responses of the experiment against the fitted responses from PLSR and PCR linear correlation methods for $\% \mathrm{CO}_{2}$ outputs.

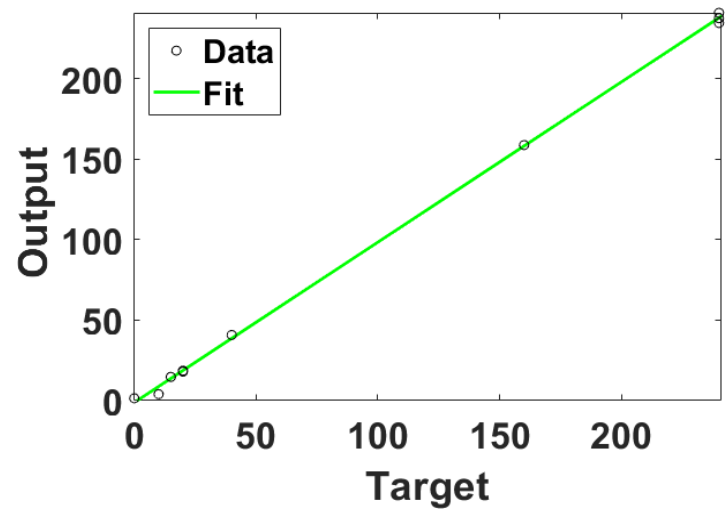

Figure 8. The experimental target samples against the output from the trained neural network for the sensor design 'holes'.

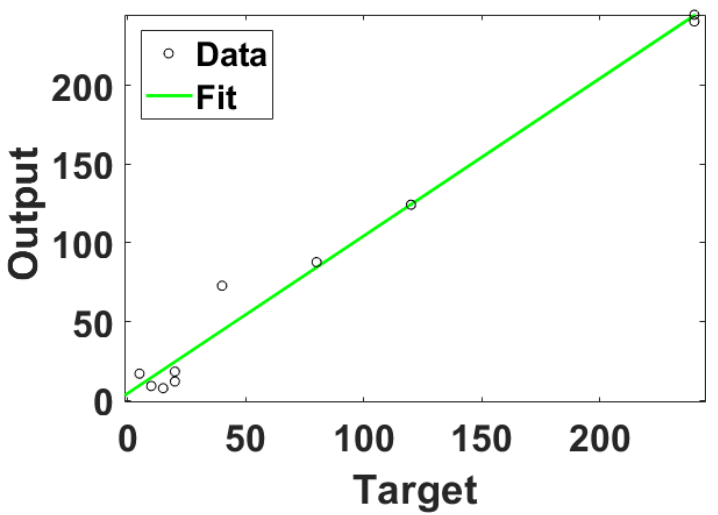

Figure 9. The experimental target samples against the output from the trained neural network for the sensor design 'no holes'.

Table 1. Table showing the results of testing the trained network 1 for the design with no holes.

TARGET

\begin{tabular}{cc} 
FLOW & $\%$ CO \\
\hline 40 & 10 \\
40 & 20 \\
160 & 0 \\
160 & 10 \\
120 & 15 \\
80 & 5 \\
& AVERAGE
\end{tabular}

OUTPUT

\begin{tabular}{|cc|} 
Flow (SCCM) & \% CO2 \\
\hline 41.95 & 9.55 \\
57.44 & 19.37 \\
160.22 & 1.46 \\
161.03 & 10.79 \\
114.92 & 10.39 \\
84.3538 & 9.33 \\
- & -
\end{tabular}

\% FS ERROR

\begin{tabular}{cc} 
Flow & \% CO2 \\
\hline 0.81 & 0.45 \\
7.27 & 0.63 \\
0.1 & 1.46 \\
0.43 & 0.79 \\
2.12 & 4.61 \\
1.81 & 4.33 \\
$\mathbf{2 . 0 9}$ & $\mathbf{2 . 0 4 5}$
\end{tabular}

Table 2. Table showing the results of testing the trained network 1 for the design with holes.

\begin{tabular}{|c|c|c|c|c|c|}
\hline \multicolumn{2}{|c|}{ TARGET } & \multicolumn{2}{|c|}{ OUTPUT } & \multicolumn{2}{|c|}{ \% FS ERROR } \\
\hline FLOW & $\% \mathrm{CO} 2$ & Flow (SCCM) & $\% \mathrm{CO} 2$ & Flow & $\% \mathrm{CO} 2$ \\
\hline 40 & 10 & 39.94 & 8.66 & 0.025 & 1.34 \\
\hline 40 & 20 & 39.69 & 20.03 & 0.13 & 0.03 \\
\hline 160 & 0 & 158.28 & 1.75 & 0.72 & 1.75 \\
\hline 160 & 10 & 159.89 & 9.15 & 0.046 & 0.85 \\
\hline 120 & 15 & 119.64 & 13.65 & 0.15 & 1.35 \\
\hline 80 & 5 & 79.12 & 4.37 & 0.37 & 0.63 \\
\hline \multicolumn{2}{|c|}{ AVERAGE } & - & - & 0.24 & 0.98 \\
\hline
\end{tabular}




\section{Linear Statistical Methods}

Two linear statistical methods were investigated to see whether they could adequately map the system in order to predict the outputs for flow and $\mathrm{CO}_{2}$. Initially, 3 PLS components were calculated and it is shown that using 2 or 3 components accounts for $99 \%$ of the variance shown in the inputs whereas 1 component shows $85 \%$ variance explanation. Therefore, it is known that there is no benefit of using 3 components and 2 components are used for the rest of the analysis. Using two components for both PLSR and PCR, the data of the actual response is plotted against the data of the fitted response for the output of flow rate (Figure 6) and $\% \mathrm{CO}_{2}$ (Figure 7). Both methods show a close relationship between the experimental outputs and those estimated by the linear methods for flow rate, meaning a good prediction of flow rate can be achieved by implementing these methods. The R-Squared value for PLSR is 0.9938 and is 0.9934 for PCR, resulting in neither method being superior. Interestingly, Figure 7 shows that neither method can accurately predict the response of $\mathrm{CO}_{2}$ with $\mathrm{R}$-squared values of 0.6436 and 0.6179 for PLSR and PCR, respectively. This suggests that the mapping is nonlinear, i.e. the principle of linear superposition does not apply, which is likely due to the strong effect from flow that is highly different for all three resistors. It is for this reason that the following investigation of using artificial neural networks is carried out.

\section{Implementation of Artificial Neural Network}

An artificial neural network has been employed to decouple the closely related parameters of flow rate and thermal conductivity, which could not be achieved through linear statistical methods. The splitting of data method known as 'Training-and-test' was used due to its extensive use for classification and discrimination problems. This method divides the available data into two groups. The first group is used to train the data using the back-propagation learning algorithm whilst the second group is used to test the trained data. In our case, 30 samples of the data were used as the training set whilst the remaining 6 were used to test the network. The Bayesian Regularization training algorithm was used due to its ability at good generalization for small noisy datasets, with the training stopping according to the adaptive weight minimization. After training the network, the sensor with holes showed a quicker convergence using 541 iterations as opposed to 1021 for the no holes design. The 6 extra samples were run through the trained network as testing data. The Mean Squared Error (MSE) is the average squared difference between the outputs from the tested data through the network and the targets. The device with membrane holes showed an average MSE of 7.28E-5, whereas the device without membrane holes shows an average MSE of 1.13E-2. This shows an increase of over 150 times, providing strong evidence that the membrane holes provide a superior response for enhanced gas discrimination. In addition to this,
Figure 8 and Figure 9 show the target of these samples plotted against the output of the trained neural net. Regression $R$ shows the correlation between these outputs and targets and the values are 0.99973 and 0.99195 for the sensor designs with holes and no holes, respectively. This once again shows the added benefit of have membrane holes. Table $1 \&$ Table 2 show the results of 6 samples used for testing the trained network for the designs with no holes and holes respectively. Here the network provided a flow rate and percentage $\mathrm{CO}_{2}$ for previously unseen $\mathrm{CO}_{2}$ concentration and/or flow rates. A Full Scale (FS) percentage error has been calculated via the difference between the target and network output as a percentage of the full reading range (i.e. 240 SCCM). Firstly, it is shown that the neural network successfully de-couples the two parameters. In addition to this, the addition of isolating holes in the membrane has multiplied the accuracy of flow measurement by approximately 10 times and the percentage $\mathrm{CO}_{2}$ by over 2 times.

Although discrimination has been displayed, there are many aspects that need to be investigated that can further optimize this sensing solution. The network is trained on relatively few data samples, using more could increase the accuracy and an optimum training set size should be investigated, i.e. decreasing the step size for percentage $\mathrm{CO}_{2}$ or flow rate measurements. In addition to this, optimum network architecture needs to be investigated, such as the number of hidden layers, neurons in these layers and backpropagation techniques. Heater temperature measurements would be a highly interesting parameter that could be incorporated into this post-processing in order to compensate for these variables. In future work, the system should be designed for real-time updating of the network training. This will allow an adaptive network that can be used for many different sensors.

\section{CONCLUSION}

An experimental method for applying artificial neural networks and two linear statistical methods in order to discriminate between flow rate and thermal conductivity has been described. The method uses a single CMOS MEMS thermal flow sensor that has three resistors embedded in the membrane. In addition to the resistors, there are two designs tested where one incorporates holes through the membrane that isolate the central resistor, forced enhanced interaction with the flowing fluid. It is shown that there is an increase in the ability to discriminate between different thermal conductivities even with the addition of flow. Using a threelayer network architecture, it was found that the two closely related parameters of thermal conductivity could be decoupled. In addition to this, the addition of the membrane holes increases the sensitivity towards flow rate by 10 times and the sensitivity towards thermal conductivity by 2 times. This sensor design coupled with well-known post-processing techniques will enable a new generation of multi-parameter sensing solutions. 


\section{ACKNOWLEDGMENT}

This research was partly funded through an Innovate UK project call "BreathFit" (file ref. 103383).

\section{REFERENCES}

[1] J. T. Kuo, L. Yu, and E. Meng, 'Micromachined thermal flow sensors-A review', Micromachines, vol. 3, no. 3, pp. 550-573, 2012.

[2] J. J. Van Baar, R. J. Wiegerink, T. S. Lammerink, G. J. Krijnen, and M. Elwenspoek, 'Micromachined structures for thermal measurements of fluid and flow parameters', J. Micromechanics Microengineering, vol. 11, no. 4, p. 311, 2001.

[3] S. Kommandur, A. Mahdavifar, P. J. Hesketh, and S. Yee, 'A microbridge heater for low power gas sensing based on the 3-Omega technique', Sens. Actuators Phys., vol. 233, pp. 231-238, 2015.

[4] J. Kuntner, F. Kohl, and B. Jakoby, 'Simultaneous thermal conductivity and diffusivity sensing in liquids using a micromachined device', Sens. Actuators Phys., vol. 130, pp. 62-67, 2006.

[5] D. Cruz, J. P. Chang, S. K. Showalter, F. Gelbard, R. P. Manginell, and M. G. Blain, 'Microfabricated thermal conductivity detector for the microChemLab $^{\mathrm{TM}}$, Sens. Actuators B Chem., vol. 121, no. 2, pp. 414-422, 2007.

[6] D. Puente, F. J. Gracia, and I. Ayerdi, 'Thermal conductivity microsensor for determining the Methane Number of natural gas', Sens. Actuators B Chem., vol. 110, no. 2, pp. 181-189, 2005.

[7] A. Mahdavifar, M. Navaei, P. J. Hesketh, M. Findlay, J. R. Stetter, and G. W. Hunter, 'Transient thermal response of micro-thermal conductivity detector $(\mu \mathrm{TCD})$ for the identification of gas mixtures: An ultrafast and low power method', Microsyst. Nanoeng., vol. 1, no. 1, p. 15025, Dec. 2015, doi: 10.1038/micronano.2015.25.

[8] E. van der Wouden, J. Groenesteijn, R. Wiegerink, and J. Lötters, 'Multi parameter flow meter for on-line measurement of gas mixture composition', Micromachines, vol. 6, no. 4, pp. 452-461, 2015.

[9] C. J. Hepp, F. T. Krogmann, and G. A. Urban, 'Flow rate independent sensing of thermal conductivity in a gas stream by a thermal MEMS-sensor-Simulation and experiments', Sens. Actuators Phys., vol. 253, pp. 136$145,2017$.

[10] K. A. Aliferis, P. A. Tarantilis, P. C. Harizanis, and E. Alissandrakis, 'Botanical discrimination and classification of honey samples applying gas chromatography/mass spectrometry fingerprinting of headspace volatile compounds', Food Chem., vol. 121, no. 3, pp. 856-862, Aug. 2010, doi: 10.1016/j.foodchem.2009.12.098.

[11] C. Bur, M. Bastuck, D. Puglisi, A. Schütze, A. Lloyd Spetz, and M. Andersson, 'Discrimination and quantification of volatile organic compounds in the ppb-range with gas sensitive SiC-FETs using multivariate statistics', Sens. Actuators B Chem., vol. 214, pp. 225-233, Jul. 2015, doi: 10.1016/j.snb.2015.03.016.

[12] T. Mehmood and B. Ahmed, 'The diversity in the applications of partial least squares: an overview', Journal of Chemometrics, 01-Jan-2016. [Online]. Available:

https://onlinelibrary.wiley.com/doi/abs/10.1002/cem.2 762. [Accessed: 02-Sep-2019].

[13] J. W. Gardner, E. L. Hines, and M. Wilkinson, 'Application of artificial neural networks to an electronic olfactory system', Meas. Sci. Technol., vol. 1, no. 5, p. 446, 1990.

[14] S. Balasubramanian, S. Panigrahi, C. M. Logue, H. Gu, and M. Marchello, 'Neural networks-integrated metal oxide-based artificial olfactory system for meat spoilage identification', J. Food Eng., vol. 91, no. 1, pp. 91-98, 2009.

[15] S. R. Johnson et al., 'Identification of multiple analytes using an optical sensor array and pattern recognition neural networks', Anal. Chem., vol. 69, no. 22, pp. 4641-4648, 1997.

[16] A. Riul et al., 'Wine classification by taste sensors made from ultra-thin films and using neural networks', Sens. Actuators B Chem., vol. 98, no. 1, pp. 77-82, Mar. 2004, doi: 10.1016/j.snb.2003.09.025.

[17] G. Huyberechts, P. Szecowka, J. Roggen, and B. W. Licznerski, 'Simultaneous quantification of carbon monoxide and methane in humid air using a sensor array and an artificial neural network', Sens. Actuators B Chem., vol. 45, no. 2, pp. 123-130, 1997.

[18] D. Zhang, J. Liu, C. Jiang, A. Liu, and B. Xia, 'Quantitative detection of formaldehyde and ammonia gas via metal oxide-modified graphene-based sensor array combining with neural network model', Sens. Actuators B Chem., vol. 240, pp. 55-65, Mar. 2017, doi: 10.1016/j.snb.2016.08.085.

[19] E. L. W. Gardner et al., 'MEMS Thermal Flow Sensors- An Accuracy Investigation', IEEE Sens. J., vol. 19, no. 8, pp. 2991-2998, Apr. 2019, doi: 10.1109/JSEN.2019.2891596.

[20] T. Aguilera, J. Lozano, J. A. Paredes, F. J. Álvarez, and J. I. Suárez, 'Electronic Nose Based on Independent Component Analysis Combined with Partial Least Squares and Artificial Neural Networks for Wine Prediction', Sensors, vol. 12, no. 6, pp. 8055-8072, Jun. 2012, doi: 10.3390/s120608055.

[21] R. Lippmann, 'An introduction to computing with neural nets', IEEE ASSP Mag., vol. 4, no. 2, pp. 4-22, Apr. 1987, doi: 10.1109/MASSP.1987.1165576. 